\title{
Quantifying vertical mixing in estuaries
}

\author{
W. Rockwell Geyer • Malcolm E. Scully • \\ David K. Ralston
}

Received: 9 April 2008 / Accepted: 29 September 2008 / Published online: 21 October 2008

(C) The Author(s) 2008. This article is published with open access at Springerlink.com

\begin{abstract}
Estuarine turbulence is notable in that both the dissipation rate and the buoyancy frequency extend to much higher values than in other natural environments. The high dissipation rates lead to a distinct inertial subrange in the velocity and scalar spectra, which can be exploited for quantifying the turbulence quantities. However, high buoyancy frequencies lead to small Ozmidov scales, which require high sampling rates and small spatial aperture to resolve the turbulent fluxes. A set of observations in a highly stratified estuary demonstrate the effectiveness of a vessel-mounted turbulence array for resolving turbulent processes, and for relating the turbulence to the forcing by the Reynolds-averaged flow. The observations focus on the ebb, when most of the buoyancy flux occurs. Three stages of mixing are observed: (1) intermittent and localized but intense shear instability during the early ebb; (2) continuous and relatively homogeneous shear-induced mixing during the mid-ebb, and weakly stratified, boundary-layer mixing during the late ebb. The mixing efficiency as quantified by the flux Richardson number $R f$ was frequently observed to be higher than the canonical value of 0.15 from Osborn (J Phys Oceanogr 10:83-89, 1980). The high efficiency may be linked to the temporal-spatial evolution of shear instabilities.
\end{abstract}

Keywords Turbulence $\cdot$ Estuaries $\cdot$ Shear instability $\cdot$ Buoyancy flux

\section{Introduction}

Vertical mixing is the most important process affecting the dynamics of estuaries, as it determines the exchange flow, the stratification, residence time and distributions of waterborne material. The classic formulations for estuarine dynamics, including Pritchard [17], Hansen

W. R. Geyer $(\varangle) \cdot$ D. K. Ralston

Applied Ocean Physics and Engineering, Woods Hole Oceanographic Institution,

Woods Hole, MA 02543, USA

e-mail: rgeyer@whoi.edu

M. E. Scully

Center for Coastal Physical Oceanography, Old Dominion University, Norfolk, VA 23529, USA 
and Rattray [8] and Chatwin [1] all parameterize the role of mixing with eddy coefficients, which depend on the strength of the tidal velocity. Many investigators have attempted to quantify estuarine turbulence with direct measurements, either of the turbulent fluxes of momentum $[6,23,25]$, the dissipation of turbulent kinetic energy $[16,19]$, and in one case, turbulent buoyancy flux [3].

The intensity of turbulence in estuaries is high compared with lakes and the ocean. High stresses and high shear rates lead to high values of turbulent kinetic energy production

$$
P=-\overline{u^{\prime} w^{\prime}} \frac{\partial \bar{u}}{\partial z}
$$

where $\overline{u^{\prime} w^{\prime}}$ is the Reynolds stress and $\partial \bar{u} / \partial z$ is the Reynolds-averaged vertical shear. This leads to turbulent kinetic energy dissipation rates $\varepsilon$ that can reach values greater than $10^{-4} \mathrm{~W} \mathrm{~kg}^{-1}$ in estuarine environments $[16,25]$. This value exceeds typical oceanic turbulence by three to four orders of magnitude [7]. The stratification in estuaries, as quantified by the buoyancy frequency $N=(g / \rho \partial \rho / \partial z)^{1 / 2}$ is also much greater than in oceanic environments due to the strong forcing by freshwater discharge. Partially mixed estuaries have typical values of $N=0.05-0.1 \mathrm{~s}^{-1}$, whereas salt wedge estuaries may have values of up to $0.3 \mathrm{~s}^{-1}$.

A comparison of the conditions in estuaries with other environments can be represented on a plot of dissipation rate versus buoyancy frequency (Fig. 1). Estuarine turbulence occurs across the top of the diagram, and oceanic turbulence lies in the lower left (low values of both

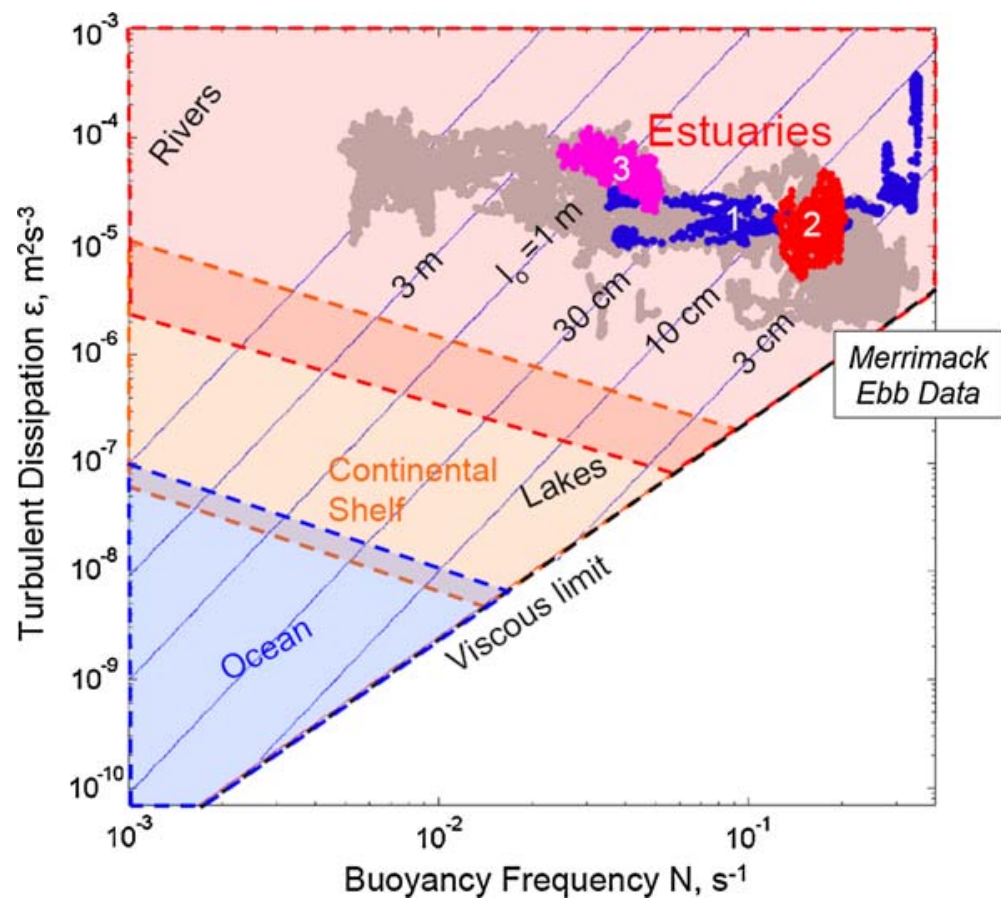

Fig. 1 Parameter space of oceanic and estuarine turbulence, based on stratification (x-axis) and kinetic energy dissipation (y-axis). The Ozmidov scale is shown in blue contours. The data from the Merrimack observations is indicated by dots. The colored dots correspond to the first, second and third observation intervals as indicated, and the gray dots correspond to the other ebb observations 
$\varepsilon$ and $N$ ). Laboratory studies of turbulence (e.g., [18]) indicate that active turbulence cannot be maintained when $\varepsilon / v N^{2}<20$ (where $v$ is the molecular viscosity), the region occupying the lower right hand side of the diagram. Interestingly, turbulence in the open ocean as well as on continental shelves is often close to this viscous threshold [7,11]. For turbulence close to the viscous threshold, the inertial subrange of spectral energy transfer is limited or absent, but for higher values of $\varepsilon / v N^{2}$, i.e., moving up and to the left in Fig. 1, the inertial subrange becomes well developed. In these conditions, $\varepsilon$ as well as the scalar variance dissipation rate $\chi$ can be estimated based on the height of the inertial subrange [20].

The Ozmidov scale $L_{\mathrm{o}}=\left(\varepsilon / N^{3}\right)^{1 / 2}$ represents the scale at which buoyancy limits the size of eddies [2]. The wide range of stratification conditions in estuaries leads to a wide range in $L_{\mathrm{o}}$, from a few $\mathrm{cm}$ in highly stratified conditions to many meters in weak stratification (Fig. 1). The small scales of the turbulence in highly stratified conditions present a challenge for measurement of turbulence by requiring small sampling volumes and high sampling frequencies to resolve the eddies. The ability to resolve the eddies is particularly important for covariance estimates of the stress and buoyancy flux. For example, the estimation of stress using an acoustic Doppler current profiler [23] would be ineffective in flows in which the eddy size is smaller than the sampling volume of the instrument, in the range of $0.25-1 \mathrm{~m}$.

This paper presents direct measurements of turbulent momentum and salt fluxes from an instrumented Mobile Array for Sensing Turbulence (the MAST) in an estuary that exhibits extreme variability in stratification and forcing conditions. The location of these data on the $\varepsilon-N$ diagram are indicated by the dots in Fig. 1. As expected for estuarine data, they fall in the upper right quadrant of the diagram-high dissipation rates as well as high buoyancy frequencies. Thus they should generally have a well developed inertial subrange, but also note that the Ozmidov scale extends from several $\mathrm{cm}$ to several $\mathrm{m}$, due mainly to the large variation of stratification. The purpose of the paper is to evaluate the effectiveness of this new approach to quantifying estuarine turbulence based on these observations, and to make some inferences as to the nature and driving mechanisms of the turbulence under varying forcing conditions. In particular, the study highlights the importance of the turbulence produced by both shear layers and boundary layers in strongly stratified estuaries.

\section{Methods}

\subsection{The measurement array for sensing turbulence (MAST)}

The MAST is a 10-m long spar attached to the side of a research vessel (Fig. 2) that provides a rigid platform for obtaining turbulence measurements. Turbulence sensors are affixed to brackets at six levels along the spar. Each bracket includes a $5 \mathrm{MHz}$ Acoustic Doppler velocimeters (Sontek Ocean ADVs) and a micro-conductivity sensors (Seabird SBE-7's). The effective sampling volume of each ADV is approximately $1 \mathrm{~cm}$, and the sampling rate is $25 \mathrm{~Hz}$. The sampling volume of each SBE-7 is $3 \mathrm{~cm}$, and its sampling rate is $200 \mathrm{~Hz}$. The sampling volumes of the conductivity cell and the ADV are only offset by $2 \mathrm{~cm}$ in the alongflow direction, allowing direct covariance estimates of the vertical salt flux $\overline{s^{\prime} w^{\prime}}$ (based on the dominance of salinity in affecting the conductivity variations in estuaries). The vertical density structure of the water column is resolved with conductivity-temperature sensors (RBR XR-620), sampling at 6 Hz. Pressure is measured at 3 of the RBRs. A six-component inertial sensor is affixed to the MAST, sampling at $10 \mathrm{~Hz}$. An altimeter mounted at the bottom of the MAST provides the distance to the bottom. The MAST can be deployed at angles ranging from $15-60^{\circ}$ from vertical, depending on the water depth. 


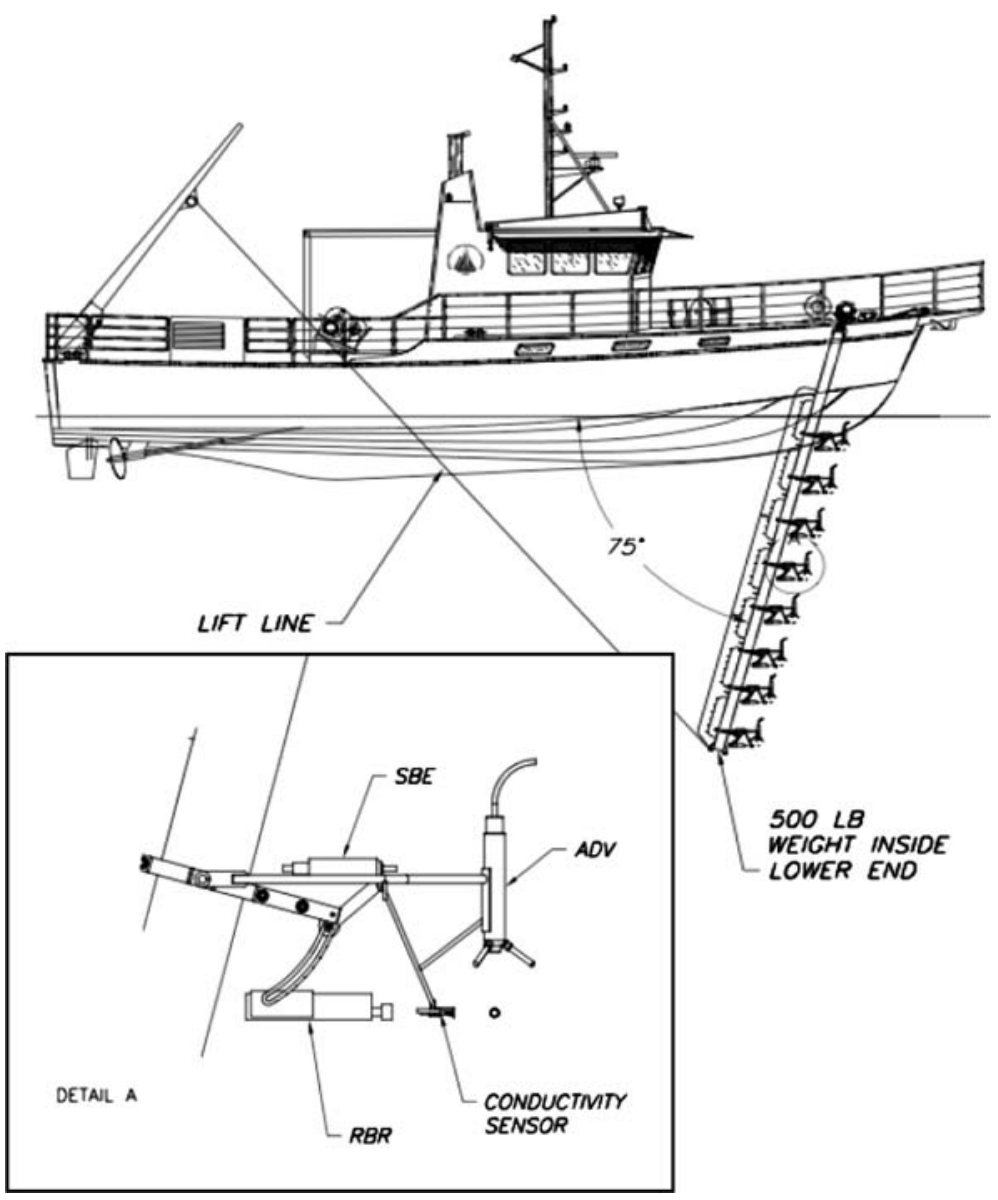

Fig. 2 Schematic of the MAST on the research vessel Tioga. For the Merrimack observations, there were only six sets of sensors. Inset shows the sensor configuration

Unlike the intermittent samples of vertical profiling, the MAST provides a temporally continuous record of mixing at multiple depths. The continuous time-series improve turbulence statistics, capture intermittent turbulent events, and provide a statistical measure of the gradient Richardson number $\left(R i=-(g / \rho) \partial \rho / \partial z /|\partial \overrightarrow{\mathbf{u}} / \partial z|^{2}\right.$, where $|\partial \overrightarrow{\mathbf{u}} / \partial z|$ is the magnitude of the vertical shear in horizontal velocity) as it varies due to changes in the Reynolds averaged flow, internal waves, and topography (with underway measurements). The advantage of the MAST over fixed boundary-layer tripods is that it extends to the upper water column where the buoyancy flux is greatest.

\subsection{Estimation of turbulence quantities}

Direct covariance estimates of momentum flux $\overline{u^{\prime} w^{\prime}}$ and salt flux $\overline{s^{\prime} w^{\prime}}$ were obtained by applying the Taylor frozen-turbulence hypothesis and computing wave number cospectra of the relevant fluctuations. Because of the dominant influence of salinity on the density in these estuarine environments, the vertical buoyancy flux was almost entirely due to fluxes of 
salinity, with heat flux being a minor contributor. Therefore, the vertical buoyancy flux could be directly estimated from the salt flux as

$$
B=\beta g \overline{s^{\prime} w^{\prime}}
$$

where $\beta=\rho^{-1} \partial \rho / \partial s$. Estimates of the energy and scalar dissipation rates $\varepsilon$ and $\chi$ were obtained from inertial-range spectra of the vertical velocity and conductivity spectra, respectively, consistent with previous published methods (e.g., [20]). The spectral form for the inertial subrange of the $w^{\prime}$ spectrum is

$$
S_{w}=a_{\mathrm{o}} \varepsilon^{2 / 3} k^{-5 / 3}
$$

where $a_{\mathrm{o}}$ is the Kolmogorov constant for velocity and $k$ is the horizontal wavenumber. Similarly, the scalar variance spectrum is of the form

$$
S_{\rho}=b_{\mathrm{o}} \chi \varepsilon^{-1 / 3} k^{-5 / 3}
$$

where $b_{\mathrm{o}}$ is the Kolmogorov constant for scalar variance and $\chi$ is the dissipation rate of scalar variance. The values for $a_{\mathrm{o}}$ and $b_{\mathrm{o}}$ were 0.68 and 0.40 , respectively, based on the formulation by Shaw et al. [20].

The inertial subrange is expected to extend roughly from the wavenumber associated with the integral turbulent length scale to the Kolmogorov wavenumber $\left(k_{\mathrm{d}}\right)$, where viscous effects begin to dominate. In the application of (3) and (4), the low wavenumber cutoff for the inertial subrange was determined from inverse of the Ozmidov scale or the inverse of the boundary layer scale $(1 / \kappa z)$, which ever was larger. Additionally, it was required that the low wavenumber cutoff have an associated frequency that was greater the $1 \mathrm{~Hz}$, to avoid any potential contamination due to surface gravity waves. The high wavenumber cutoff for the inertial subrange was estimated conservatively as $0.05 k_{\mathrm{d}}$ (where $k_{\mathrm{d}}$ is the Kolmogorov wavenumber $\left.k_{\mathrm{d}}=\left(\varepsilon / v^{3}\right)^{1 / 4}\right)$. Because estimates of both the Ozmidov and Kolmogorov wave numbers require knowledge of the dissipation rate, an iterative procedure was used in determining the limits of the inertial subrange for the calculation of dissipation. Using this methodology, a finite inertial subrange was present in all but the lowest energy conditions.

At high energy levels typical of boundary layers and weak to moderate stratification, the MAST provides direct estimates of Reynolds stress and buoyancy flux, as well as the dissipation rates of turbulent kinetic energy and scalar variance. Examples of spectra of vertical velocity variance and density variance are shown in Fig. 3 for selected observations in the Merrimack estuary. Note that the inertial subrange extends for more than a decade in these examples, providing reliable estimates of $\varepsilon$ and $\chi$ from the vertical velocity and conductivity spectra, respectively. The right-hand panel of Fig. 3 illustrates the integrated co-spectrum of vertical velocity and density, as expressed as buoyancy flux. The integral extends from the Nyquist wavenumber (approximately 100-200 rad/m) through the energy-containing scales of the turbulence. For the examples shown, the maximum contribution to the buoyancy flux was at scales of $0.1-1 \mathrm{~m}$.

Turbulent flux calculations using direct integration of the co-spectra were not always possible because of the presence of surface gravity waves. Additionally, internal wave energy may be present at frequencies lower than the buoyancy frequency. To avoid potential contamination from either source, we evaluated the measured cospectra with the theoretical model of Kaimal etal. [9] Following the methods proposed by Gerbi et al. [4], we fit the theoretical model of Kaimal etal. [9] to the observed cospectra, omitting the energy in frequencies where surface gravity wave energy was observed $(0.15<f<0.85 \mathrm{~Hz})$, as well as energy at wavenumbers where internal energy may be present $(k<2 \pi N / U)$. The peak of the 

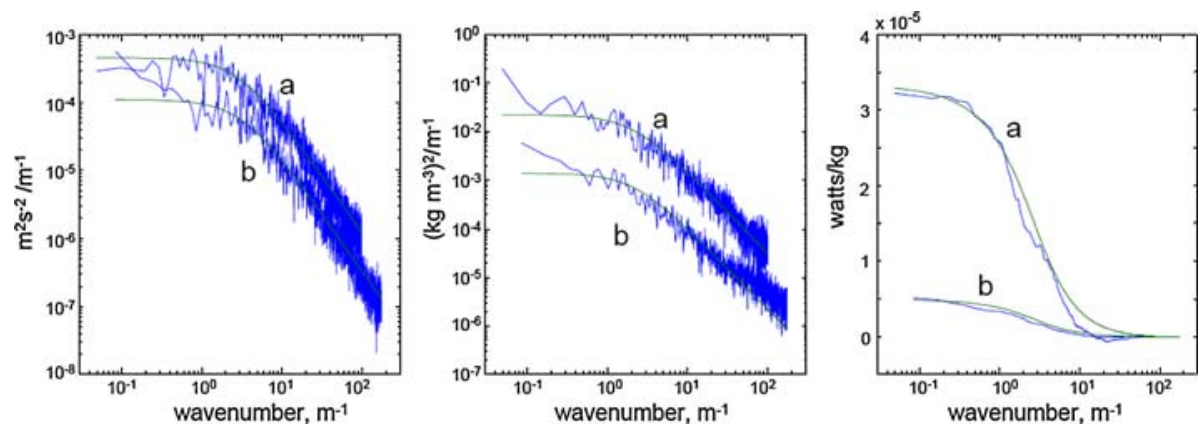

Fig. 3 Spectra of vertical velocity variance from ADV (left panel), density variance from micro-conductivity (middle) and buoyancy flux (right) based on the covariance $(g / \rho) \overline{\rho^{\prime} w^{\prime}}$ for two observation periods (a, b). The density variance was calculated based on the conductivity variance, using the measured T-S relationship to provide a continuous estimate of $\partial \rho / \partial c$. The green lines show the Kaimal et al. [9] fits to the spectra

normalized cospectra was generally at wavenumbers significantly higher than those possibly influenced by internal waves. Additionally, because of the limited fetch in the Merrimack, surface gravity waves were only present in a very narrow frequency band. As a result, the peak of the cospectra were almost always well resolved using this method. Examples of the cospectral fits are shown in Fig. 3c for conditions when wave energy was minimal.

In contrast to the 5-min estimates of cospectra, we found reliable inertial subrange estimates of $\varepsilon$ and $\chi$ could be obtained from significantly shorter time periods ( $\sim 1 \mathrm{~min}$ ). This allows higher temporal resolution estimates of buoyancy flux using the Osborn and Cox [14] approach of relating $\chi$ to $B$ via the assumption that scalar variance production and dissipation are in balance:

$$
\overline{\rho^{\prime} w^{\prime}} \frac{\partial \rho}{\partial z}=\frac{\chi}{2}
$$

Evidence for the validity of this assumption is shown in Fig. 4, where the buoyancy flux estimated from the Osborn-Cox model is compared to direct estimates based on the theoretical cospectral fits. Although there is some scatter (based on 5-min averages), the two methods of estimating buoyancy flux are well correlated with over $76 \%$ of the observation balancing to within a factor of 2, providing support for the use of the Osborn-Cox model to estimate buoyancy flux.

\section{Field observations}

Measurements were obtained in the Merrimack estuary (Fig. 5) during moderate river outflow conditions in May, 2007. The Merrimack is a highly stratified, salt-wedge type estuary with tidal currents of approximately $1 \mathrm{~m} / \mathrm{s}$. The measurements focused on a region of strong horizontal density gradient that occurred in a region of channel expansion during the ebbing tide. The MAST was deployed from an anchored research vessel in 6-8 $\mathrm{m}$ water depth.

During the early ebb, the pycnocline was in the upper part of the water column, with strong outflow of nearly fresh water at the surface and a strong shear zone across the pycnocline (Fig. 6). The near-bottom velocity was weak, and bottom stress had no influence on the turbulence within the pycnocline. During the middle ebb, the pycnocline broadened to extend through the water column, and the velocity shear commensurately broadened. Near-bottom 


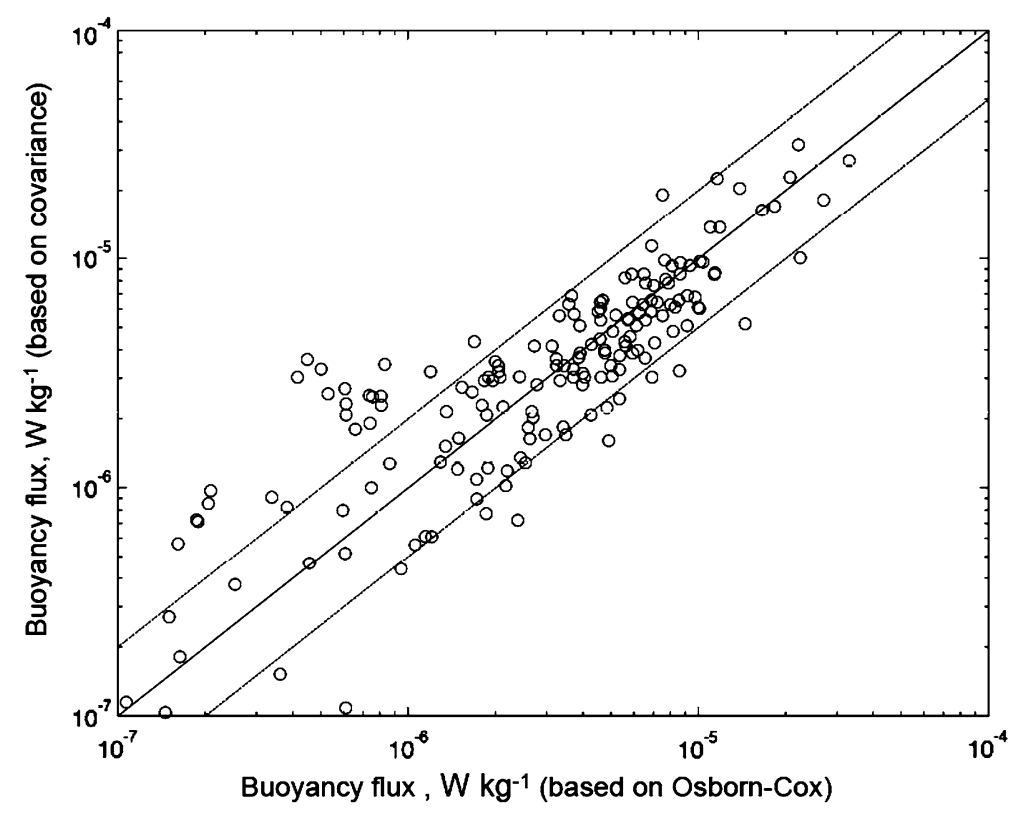

Fig. 4 Buoyancy flux $\left(\mathrm{W} \mathrm{kg}^{-1}\right)$ estimated from the Osborn and Cox [14] method compared with direct estimates of buoyancy flux from covariance $(g / \rho) \overline{\rho^{\prime} w^{\prime}}$ based on 5-min average data during the 2007 Merrimack River study

velocity was still weak. During late ebb, salt was entirely flushed from this portion of the estuary, near-bottom currents increased markedly, and boundary-layer turbulence dominated through the water column.

The MAST measurements at the early, middle and late phases of the ebb show distinct changes in the characteristics of the turbulence. During the early ebb, a patch of intense turbulence occurred in the upper pycnocline (Fig. 7), just following a slight drop in the pycnocline elevation. This patch is identifiable in the density record by high-frequency, large-amplitude fluctuations of the density interface around $1.5 \mathrm{~m}$ depth. A close look at these disturbances indicates that they were dominated by steep internal waves, with periods of roughly $4 \mathrm{~s}$ and amplitudes of $10-20 \mathrm{~cm}$. Although the vertical structure of the waves was not well resolved by the sensor array, there appeared to be a vertical phase shift in the wave structure consistent with shear instability [21]. Instantaneous values of $R i$ were between 0.5 and 1 before the mixing event (Fig. 7, third panel), and were maintained at a near-constant value of 0.35 during much of the period of mixing. The local value of $R i$ may have been below 0.25 , but the spacing between sensors of $0.7 \mathrm{~m}$ did not fully resolved the vertical gradients (see Sect. 4).

The highest dissipation rate was recorded at the turbulence sensors at 1.7-m depth (Fig. 3, second panel, Table 1), with several peaks exceeding $3 \times 10^{-4} \mathrm{~W} \mathrm{~kg}^{-1}$ during the period of density fluctuations. Prior to and following this event, the dissipation rate was an order of magnitude lower. Buoyancy flux (as determined by Eq.5) also showed several peaks at the 1.8 -m depth level, roughly tracking the variations in $\varepsilon$. The stress estimates were too noisy to be resolved during this period due apparently to non-stationarity associated with the internal wave variations, so we estimated the flux Richardson number as $R f \cong B /(\varepsilon+B)$. It was found to be 0.26 at $1.8 \mathrm{~m}$ based on the average buoyancy flux and average dissipation over the 10-min interval (see Table 1). 

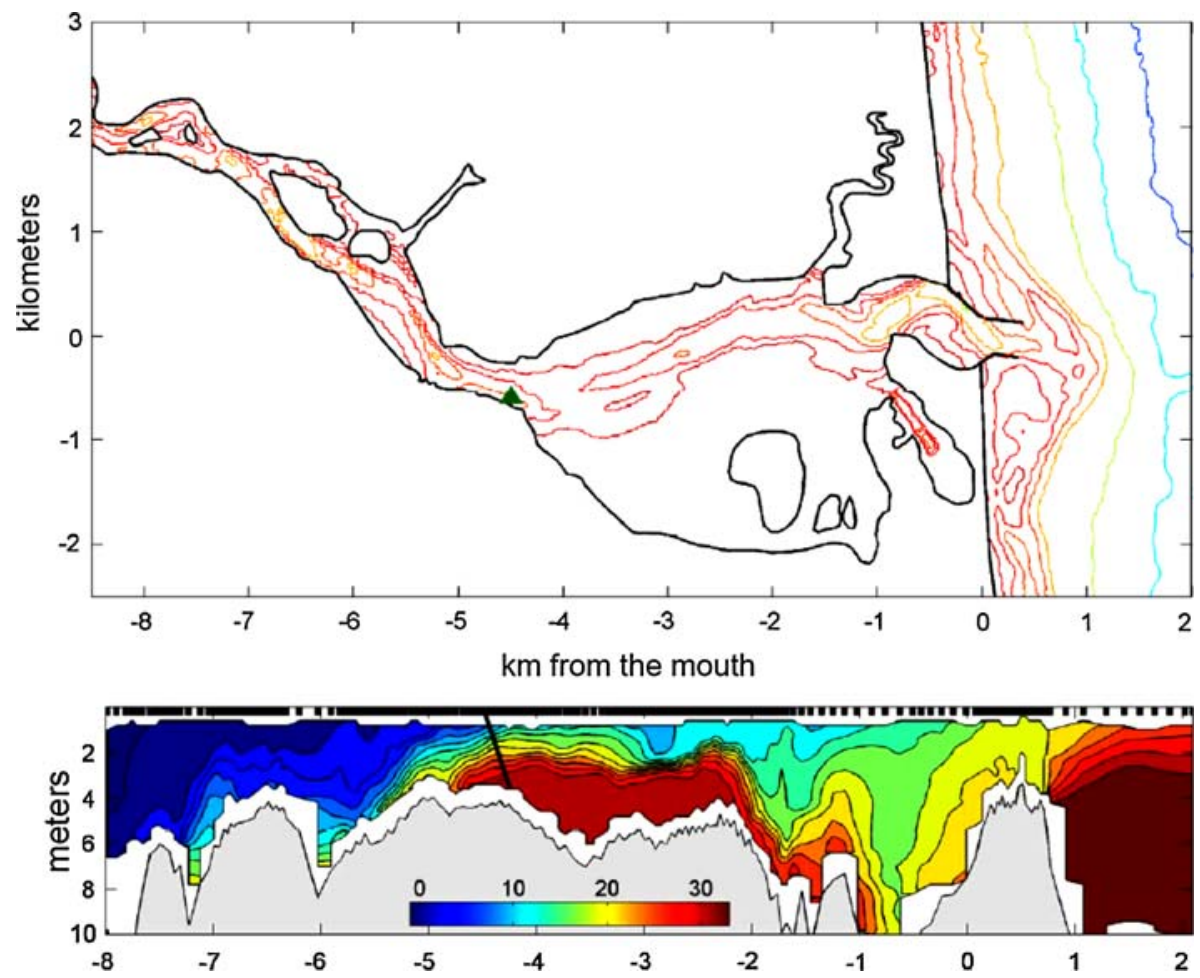

Fig. 5 The Merrimack estuary. Upper panel: bathymetry contours, with 2-m contours. Lower panel: salinity contours during the early to mid-ebb, based on a shipboard survey on May 18, 2005. The location of the 2007 measurements are shown with the green triangle (upper panel) and the schematic of the MAST at $\mathrm{km}-4.3$ (lower panel)

Fig. 6 Salinity (from profiling CTD) and velocity profiles (from shipboard ADCP) during the early, middle and late ebb of May 13, 2007. These data were obtained by a different vessel from the one deploying the MAST, so the depths are slightly different from the MAST observations
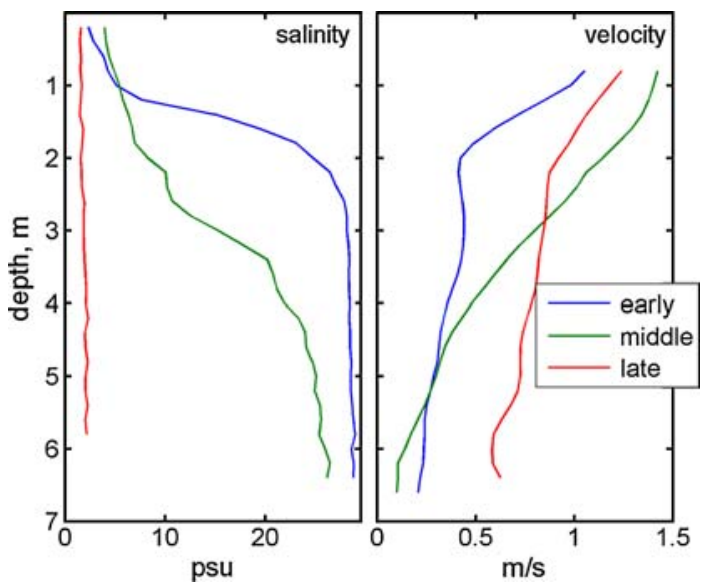

During the middle ebb (Fig. 8), the density gradient extended through the entire water column, and density fluctuations were evident but not as intense as the previous event. Dissipation rates were $1-3 \times 10^{-5} \mathrm{~W} \mathrm{~kg}^{-1}$, approximately an order of magnitude lower than 


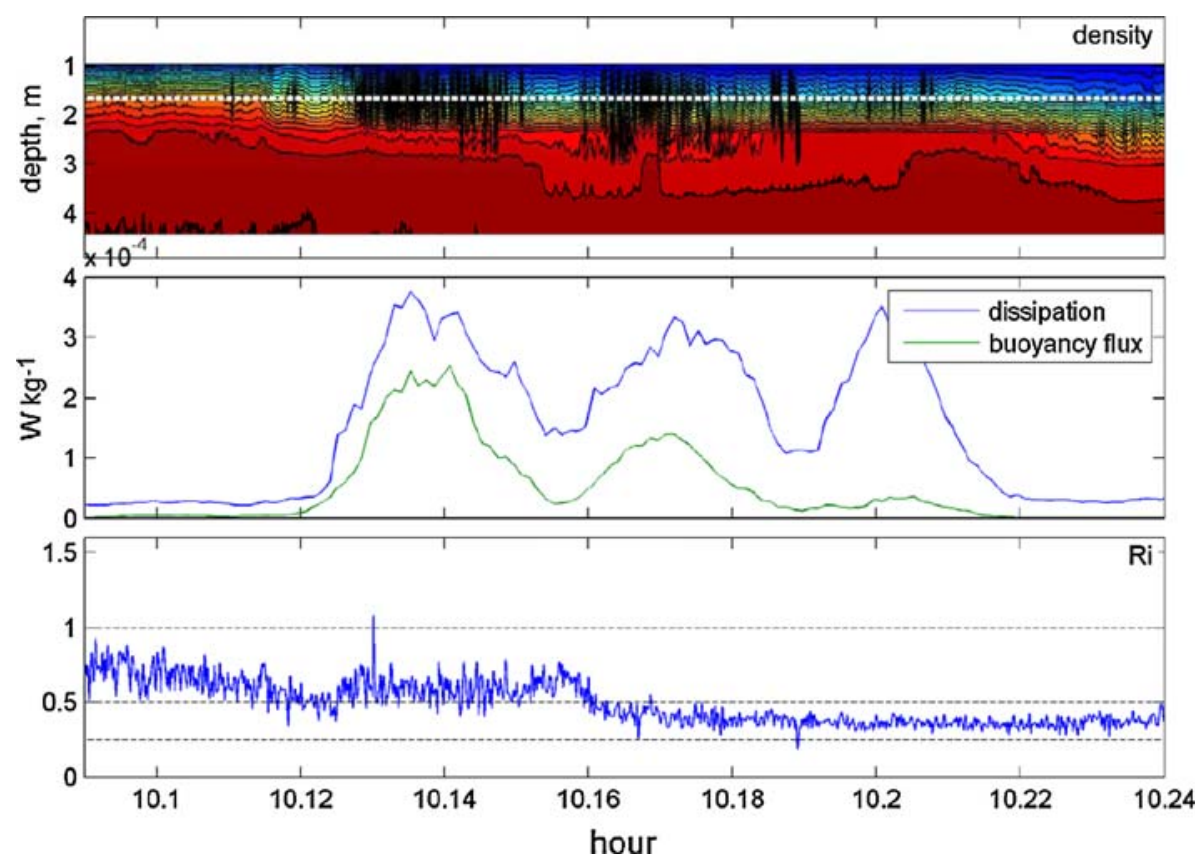

Fig. 7 Density contours (upper panel), dissipation and buoyancy flux (middle panel) and Ri (lower panel) for the early-ebb period (water depth: $7.6 \mathrm{~m}$ ). Contour interval for density is $1 \mathrm{~kg} \mathrm{~m}^{-3}$. The white line indicates the level at which the displayed quantities were measured

Table 1 Turbulence parameters during the first observation interval: early ebb

\begin{tabular}{lcllll}
\hline Depth (m) & $\mathbf{1 . 7}$ & 2.4 & 3.0 & 3.7 & 4.4 \\
$\varepsilon \times 10^{5}\left(\mathrm{~W} \mathrm{~kg}^{-1}\right)$ & $\mathbf{1 4 . 3}$ & 3.4 & 1.7 & 1.4 & 2.5 \\
$B \times 10^{5}\left(\mathrm{~W} \mathrm{~kg}^{-1}\right)$ & $\mathbf{4 . 9}$ & 1.4 & 0.9 & $*$ & 0.1 \\
$R f$ & $\mathbf{0 . 2 6}$ & 0.29 & 0.05 & $*$ & 0.06 \\
$R i$ & $\mathbf{0 . 3 3}$ & 0.63 & 2.2 & 1.3 & 0.06 \\
$N\left(\mathrm{~s}^{-1}\right)$ & $\mathbf{0 . 3 4}$ & 0.27 & 0.12 & 0.07 & 0.07 \\
$L_{\mathrm{O}}(\mathrm{m})$ & $\mathbf{0 . 0 5}$ & 0.04 & 0.11 & 0.26 & 0.40 \\
Height above bottom $(\mathrm{m})$ & $\mathbf{6 . 0}$ & 5.3 & 4.6 & 3.9 & 3.2 \\
\hline
\end{tabular}

The bold-faced column corresponds with the data plotted in Fig. 7

* Indicates data rejected due to sensor fouling

the early-ebb event, but the turbulence was more uniformly distributed through the water column. The highest dissipation rates were at the bottommost sensor (Table 2), but $\varepsilon$ was nearly constant in the vertical, contrasting the highly inhomogeneous conditions of the first interval. Buoyancy flux consistently tracked the dissipation rate, with average efficiency $R f$ $\sim 0.3$ except at the top-most sensor, at which it was lower, consistent with lower $R i$ at that level (Table 2). These values of $R f$ are slightly higher than the canonical value of 0.15 [13]. Possible reasons for these high values of $R f$ are discussed in the next section.

During the late ebb (Fig.9), the vertical density structure was weak, but there was still significant buoyancy flux. Vertical excursions of density were large, but the density variations were slight. The dissipation rate was highest near the bottom, monotonically decreasing 


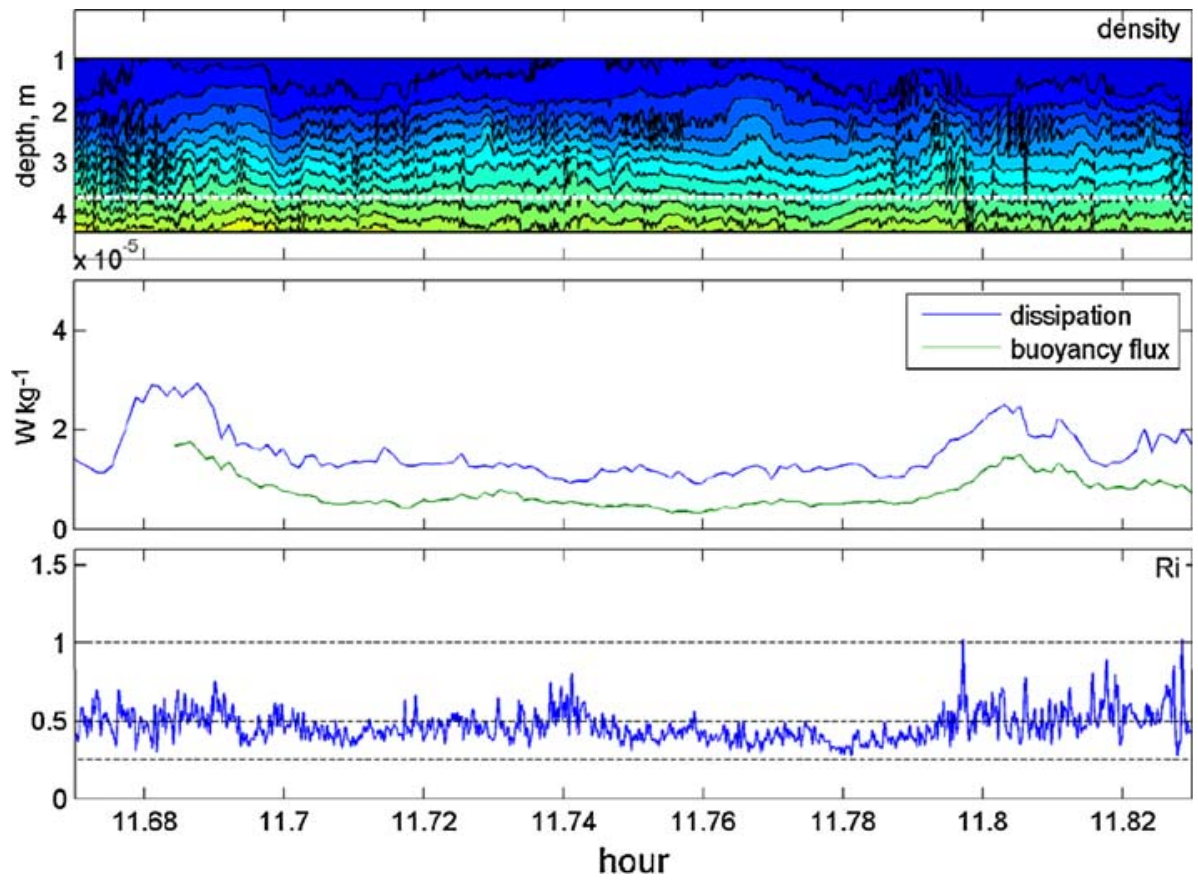

Fig. 8 Same quantities as Fig. 7 for the middle ebb interval (water depth $6.5 \mathrm{~m}$ )

Table 2 Turbulence parameters during the second observation interval: mid-ebb

\begin{tabular}{llllll}
\hline Depth $(\mathrm{m})$ & 1.6 & 2.3 & 3.0 & $\mathbf{3 . 7}$ & 4.4 \\
$\varepsilon \times 10^{5}\left(\mathrm{~W} \mathrm{~kg}^{-1}\right)$ & 1.3 & 1.5 & 1.6 & $\mathbf{1 . 5}$ & 2.1 \\
$B \times 10^{5}\left(\mathrm{~W} \mathrm{~kg}^{-1}\right)$ & 0.2 & 0.8 & 0.5 & $\mathbf{0 . 7}$ & 1.1 \\
$R f$ & 0.11 & 0.33 & 0.25 & $\mathbf{0 . 3 2}$ & 0.35 \\
$R i$ & 0.11 & 0.46 & 0.28 & $\mathbf{0 . 3 9}$ & 0.35 \\
$N\left(\mathrm{~s}^{-1}\right)$ & 0.14 & 0.18 & 0.18 & $\mathbf{0 . 1 8}$ & 0.17 \\
$L_{\mathrm{O}}(\mathrm{m})$ & 0.07 & 0.05 & 0.05 & $\mathbf{0 . 0 5}$ & 0.07 \\
Height above bottom (m) & 4.9 & 4.2 & 3.5 & $\mathbf{2 . 9}$ & 2.2 \\
\hline
\end{tabular}

The bold-faced column corresponds to the data plotted in Fig. 8

in the vertical (Table 3), consistent with boundary-layer turbulence. Buoyancy fluxes were significantly lower, around $2 \times 10^{-5} \mathrm{~W} \mathrm{~kg}^{-1}$, with $R f \sim 0.1-0.2$ (Table 3 ) . $R i$ was persistently less than 0.25 during this period. Interestingly, the mean values of $R i$ were smaller than $R f$ during this interval, whereas the $R i$ tended to exceed $R f$ during the second interval. This difference indicates a change in the influence of fluctuations in stratification and shear between marginally unstable conditions (period 2) and persistently unstable, weakly stratified conditions (period 3). In the former, significant buoyancy fluxes occur only when $R i$ drops below its critical value, whereas in the latter, the largest buoyancy fluxes occur during periods of stronger stratification. 


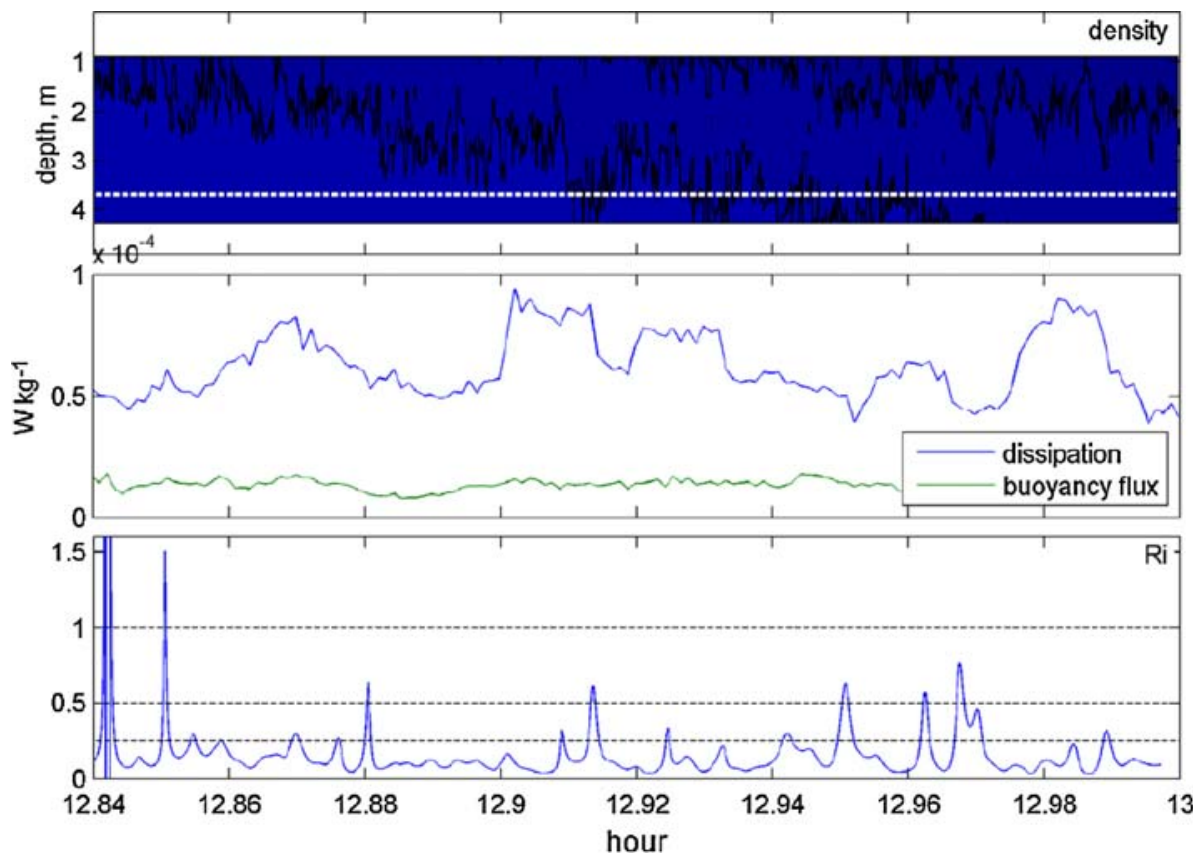

Fig. 9 Same quantities as Fig. 7 for the late ebb interval (water depth $5.9 \mathrm{~m}$ ). Density contour interval changed to $0.5 \mathrm{~kg} \mathrm{~m}^{-3}$. $R i$ was very noisy due to weak density gradients and large fluctuations in shear, so the stratification and shear were filtered at $10 \mathrm{~s}$ during this time interval to provide a more stable estimate

Table 3 Turbulence parameters during the third observation interval: late ebb

\begin{tabular}{llllll}
\hline Depth $(\mathrm{m})$ & 1.6 & 2.3 & 2.9 & $\mathbf{3 . 6}$ & 4.3 \\
$\varepsilon \times 10^{5}\left(\mathrm{~W} \mathrm{~kg}^{-1}\right)$ & 3.2 & 4.8 & 5.6 & $\mathbf{6 . 2}$ & 7.6 \\
$B \times 10^{5}\left(\mathrm{~W} \mathrm{~kg}^{-1}\right)$ & 1.1 & $*$ & 1.1 & $\mathbf{1 . 3}$ & 0.9 \\
$R f$ & 0.26 & $*$ & 0.17 & $\mathbf{0 . 1 7}$ & 0.10 \\
$R i$ & 0.02 & 0.10 & 0.06 & $\mathbf{0 . 0 6}$ & 0.05 \\
$N\left(\mathrm{~s}^{-1}\right)$ & 0.05 & 0.05 & 0.04 & $\mathbf{0 . 0 3}$ & 0.03 \\
$L_{\mathrm{o}}(\mathrm{m})$ & 0.6 & 0.7 & 1.0 & $\mathbf{1 . 3}$ & 1.6 \\
Height above bottom $(\mathrm{m})$ & 4.3 & 3.6 & 2.9 & $\mathbf{2 . 3}$ & 1.6 \\
\hline
\end{tabular}

The bold-faced column corresponds to the data plotted in Fig. 9

\section{Discussion}

\subsection{Assessment of the measurement approach}

These observations demonstrate that variations in dissipation and buoyancy flux can be resolved at timescales as short as $1 \mathrm{~min}$, and that indeed there are order-of-magnitude changes in these quantities over such time-scales. The minimum time-scale of the inertial subrange estimates of $\varepsilon$ and $B$ is set by the time interval required to obtain a statistically significant estimate of the inertial subranges of velocity and density, which was found to be on the order of $1 \mathrm{~min}$. This compares to a timescale of $5-10 \mathrm{~min}$ to obtain reliable estimates of 
buoyancy flux and Reynolds stress via direct covariance estimates. The agreement between direct measurements of buoyancy flux and inertial-subrange estimates (cf. Fig. 4) provide some confidence that the inertial method can improve temporal resolution of buoyancy flux.

\subsection{Mixing efficiency}

The distribution of $R f$ (based on 1-min averages) for the entire 4-h ebb period is shown in Fig. 10. Almost half the points indicate $R f>0.15$ (the median is 0.146 ), but only $10 \%$ of the values exceed $R f=0.35$. The low values of efficiency are associated with the weak stratification at the end of the timeseries.

These efficiency estimates are generally higher than have been reported for laboratory [18] and oceanic measurements [13], although these values are comparable to some recent atmospheric measurements [15]. Moreover, direct numerical simulations indicate that local values of efficiency vary markedly within evolving shear instabilities ([21], [22]). It is possible that the observations in this location were generally phase-locked to the early to middle phases of development of shear instabilities, in which buoyancy fluxes and mixing efficiency would be higher than their spatial averages (see discussion of mixing dynamics, below). It is also possible that the buoyancy fluxes were systematically overestimated. Our estimates of $B$ derived from $\chi$ depend upon the value of the Kolmogorov constant for scalar dissipation $b_{\mathrm{o}}$. While we use a value of 0.40 following Shaw et al. [20], other literature values range from 0.41 to as high as 2.3 [9]. A significant increase in $b_{\mathrm{o}}$ would degrade the scalar variance balance (Fig. 4), but an increase of $b_{\mathrm{o}}$ by $30 \%$ would still be consistent with that balance, and it would result in a commensurate $30 \%$ decrease in the buoyancy flux and $\Gamma$. It is also possible that internal wave variance elevated the estimate of the inertial subrange of scalar variance. The part of the spectrum that was included in this estimate should be in significantly higher

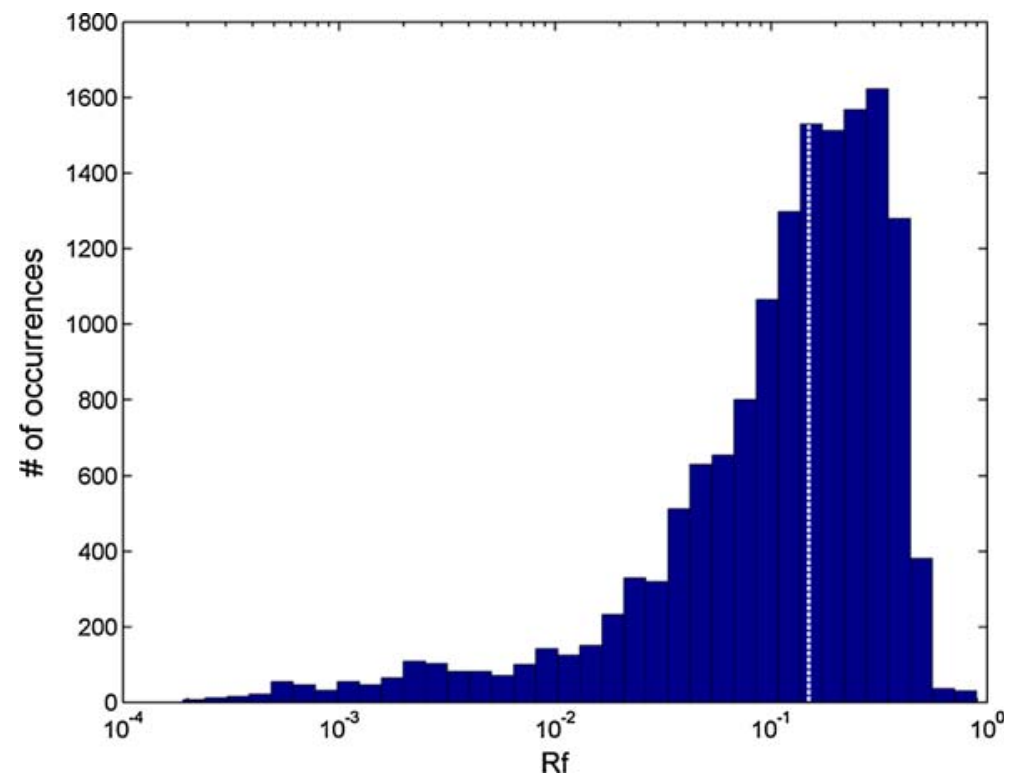

Fig. 10 Histogram of $R f$ (based on the approximation $R f \cong B /(\varepsilon+B)$ ) for the entire 4-h period of ebb observations. Each point is based on a running 1-min mean of the estimated dissipation and buoyancy flux. The dashed line is the Osborn [13] value $R f=0.15$ 
frequencies than the internal wave band, but perhaps the Doppler shifting of steep internal waves by the tidal flow contributed some wave variance into the inertial subrange band.

\subsection{Interpretation of the mixing processes}

The three intervals displayed in Figs. 6-9 show three different types of mixing conditions: an initial period of inhomogeneous, intermittent mixing; an intermediate condition of relatively persistent and homogeneous mixing close to its maximum efficiency, and finally weakly stratified, boundary-layer mixing. During the first interval of intermittent mixing, high turbulence levels occur coincident with steep, high-frequency internal waves that are likely manifestations of Kelvin-Helmholtz instabilities, as noted above. Instability requires that $R i<0.25$ [12], whereas the measured value of $R i$ was just above 0.5 when these waves first appeared. However, the vertical resolution of the sensors was $0.7 \mathrm{~m}$, and if the gradients were underresolved during the early ebb (cf. Fig. 6), $R i$ may have been overestimated. It is possible that the instabilities initiated in a region of stronger shear upstream, and the MAST observations downstream were of density and velocity profiles modified by mixing. The spatial salinity structure in Fig. 5 indicates a frontal transition zone just upstream of the MAST observations (albeit in a different year but during similar run-off and tidal conditions), so it is likely that the instabilities initiated in this frontal region.

The conditions during the second interval indicate "saturated" mixing conditions, in which $R i$ is close enough to 0.25 throughout most of the water column to maintain persistent shearinduced mixing. The average value of $R i \cong 0.35$ observed during this period is similar to those observed in laboratory studies of shear instabilities by Thorpe [24] and Koop and Browand [10] and in many field studies (e.g., [5]).

The final interval is consistent with boundary-layer mixing, as indicated by the high dissipation rates near the bottom and the roughly inverse dependence on distance from the bottom (Table 3). Stratification only slightly modifies the turbulence structure, as evidenced by the low values of $R i$ and $R f$ and the relatively large value of $L_{0}$. However buoyancy flux is still important during this period in terms of its overall contribution to mixing in the estuary, due to the elevated dissipation rates that lead to significant buoyancy fluxes even though the efficiencies are lower than the more stratified periods.

\subsection{Scaling of turbulence production}

The values of dissipation rate and buoyancy flux during the early ebb are surprisingly high, so we are obliged to consider the dynamics that would lead to these high levels of turbulence. As the surface and bottom stresses were weak during the early ebb, the most likely agent to force the shear at this time is the baroclinic density gradient. If a local balance between the baroclinic forcing and stress divergence is assumed, the turbulence production can be estimated as

$$
P=\frac{1}{4} g^{\prime} \frac{\partial h_{i}}{\partial x} \Delta u
$$

where $g^{\prime}=g \frac{\Delta \rho}{\rho} \cong 0.2 \mathrm{~m} \mathrm{~s}^{-2}$ is the reduced gravity, $\partial h_{i} / d x$ is the slope of the pycnocline, and $\Delta u \cong 1 \mathrm{~m} \mathrm{~s}^{-1}$ is the velocity difference across the pycnocline. In order to achieve a turbulence production rate commensurate with the peak dissipation rate of $3 \times 10^{-4} \mathrm{~W} \mathrm{~kg}^{-1}$ observed during the early-ebb event, the interface slope would have to be roughly 1:200. Although that is an order of magnitude higher than the average slope of the pycnocline, it 
is comparable to the steepest slope observed in the pycnocline in the frontal zone in Fig. 5. The more modest dissipation rate observed during the mid-ebb would only require a slope of 1:2000, which is consistent with the average pycnocline slope in the non-frontal areas.

During the late ebb observations, the turbulence production was no longer associated with baroclinic forcing but was driven by boundary-layer turbulence. The bottom stress was approximately $1 \mathrm{~Pa}$ (based on direct covariance estimates at the deepest sensor), and the estimated turbulence production (Eq. 1) was found to be comparable to the observed dissipation rate during this period.

\section{Conclusions}

These observations demonstrate the value of continuous turbulence measurements to identify and quantify mixing processes in the highly variable stratification conditions of estuaries. The inertial-subrange method of estimating turbulent energy and scalar variance dissipation rates is effective in the high-energy conditions of estuaries, and the continuous timeseries provide high temporal resolution of the turbulent variations. The observations in the Merrimack estuary reveal three distinct mixing regimes: (1) intense mixing associated with localized shear instability in a frontal zone during early ebb; (2) relatively homogeneous, low-intensity stratified mixing during mid-ebb; and (3) highly dissipative, boundary-layer mixing of the weakly stratified flow during the late ebb. These observations reveal higher mixing efficiencies than the canonical value of Osborn [13]. Although methodological issues cannot be ruled out, another explanation is that mixing efficiency increases locally in association with the evolution of shear instabilities, as proposed by Smyth et al. (2001).

This study does not provide a comprehensive look at estuarine mixing, as it focuses on one ebb period in a highly stratified estuary. However it does provide a surprisingly broad range of conditions in the $\varepsilon-N$ space, as revealed in Fig. 1, and this small data-set encompasses several important classes of stratified mixing processes. This type of data provides an effective test-bed for turbulence closure modeling and for refinement of turbulence parameterizations. In particular, this study highlights the importance of addressing mixing efficiency, and the possible influence of spatial evolution of shear instabilities as it may affect the relative strength of buoyancy flux and dissipation. With appropriate closure models, numerical simulations can provide generalizations of these results to estuary-scale integration of the mixing processes.

Acknowledgments The funding for this research was obtained from ONR Grant N00014-06-1-0292 and NSF Grant OCE-0729547.

Open Access This article is distributed under the terms of the Creative Commons Attribution Noncommercial License which permits any noncommercial use, distribution, and reproduction in any medium, provided the original author(s) and source are credited.

\section{References}

1. Chatwin PC (1976) Some remarks on the maintenance of the salinity distribution in estuaries. Estuar Coast Mar Sci 4:555-566. doi:10.1016/0302-3524(76)90030-X

2. Dillon TM (1982) Vertical overturns: a comparison of Thorpe and Ozmidov length scales. J Geophys Res 87:9601-9613. doi:10.1029/JC087iC12p09601

3. Gargett AE (1994) Observing turbulence with a modified acoustic Doppler current profiler. J Atmos Ocean Technol 11:1592-1610. doi:10.1175/1520-0426(1994)011<1592:OTWAMA>2.0.CO;2 
4. Gerbi GP, Trowbridge JH, Edson JB, Plueddemann AJ, Terray EA, Fredericks JJ (2008) Direct covariance measurements of momentum and heat transfer across the air-sea interface. J Phys Oceanogr 38:10541072. doi:10.1175/2007JPO3739.1

5. Geyer WR, Smith JD (1987) Shear instability in a highly stratified estuary. J Phys Oceanogr 17:1668-1679

6. Gordon CM, Dohne CF (1973) Some observations of turbulent flow in a tidal estuary. J Geophys Res 78:1971-1978. doi:10.1029/JC078i012p01971

7. Gregg MC (1984) Entropy generation in the ocean by small-scale mixing. J Phys Oceanogr 14:688-711. doi:10.1175/1520-0485(1984)014<0688:EGITOB >2.0.CO;2

8. Hansen DV, Rattray M (1965) Gravitational circulation in straits and estuaries. J Mar Res 23:104-122

9. Kaimal JC, Wyngaard JC, Izumi Y, Cote OR (1972) Spectral characteristics of surface-layer turbulence. Q J R Metab Soc 98:563-589. doi:10.1002/qj.49709841707

10. Koop CG, Browand FK (1979) Instability and turbulence in a stratified fluid with shear. J Fluid Mech 93:135-159. doi:10.1017/S0022112079001828

11. Ledwell JR, Watson AJ, Law CS (1993) Evidence for slow mixing across the pycocline from an openocean tracer-release experiment. Nature 364:701-703. doi:10.1038/364701a0

12. Miles JW (1961) On the stability of heterogeneous shear flow. J Fluid Mech 10:496-506. doi:10.1017/ S0022112061000305

13. Osborn TR (1980) Estimates of the local rate of vertical diffusion from dissipation measurements. J Phys Oceanogr 10:83-89. doi:10.1175/1520-0485(1980)010<0083:EOTLRO>2.0.CO;2

14. Osborn TR, Cox CS (1972) Oceanic fine structure. Geophys Fluid Dyn 3:321-345. doi:10.1080/ 03091927208236085

15. Pardyjak ER, Monti P, Fernando HJS (2002) Flux Richardson number measurements in stable atmospheric shear flows. J Fluid Mech 459:307-316. doi:10.1017/S0022112002008406

16. Peters H (2003) Broadly distributed and locally enhanced turbulent mixing in a tidal estuary. J Phys Oceanogr 33:1967-1977. doi:10.1175/1520-0485(2003)033<1967:BDALET>2.0.CO;2

17. Pritchard DW (1952) Salinity distribution and circulation in the Chesapeake Bay estuarine system. J Mar Res 11:106-123

18. Rohr JJ, Itsweire EC, Helland KN, Van Atta CW (1988) Growth and decay of turbulence in a stably stratified shear flow. J Fluid Mech 195:77-111. doi:10.1017/S0022112088002332

19. Seim HE, Gregg MC (1994) Detailed observations of a naturally occurring shear instability. J Geophys Res 100:4943-4958. doi:10.1029/94JC03199

20. Shaw WJ, Trowbridge JH, Williams AJ III (2001) Budgets of turbulent kinetic energy and scalar variance in the continental shelf bottom boundary layer. J Geophys Res 106:9551-9564. doi:10.1029/2000JC000240

21. Smyth WD, Moum JN (2000) Length scales of turbulence in stably stratified mixing layers. Phys Fluids 12:1327-1342. doi:10.1063/1.870385

22. Smyth WD, Moum JN, Caldwell DR (2001) The efficiency of mixing in turbulent patches:inferences from direct numerical simulations and microstructure observations. J Phys Oceanogr 31:1969-1992

23. Stacey MT, Monismith SG, Burau JR (1999) Observations of turbulence in a partially stratified estuary. J Phys Oceanogr 29:1950-1970. doi:10.1175/1520-0485(1999)029<1950:OOTIAP>2.0.CO;2

24. Thorpe SA (1973) Experiments on instability and turbulence in a stratified shear flow. J Fluid Mech 61(4):731-751. doi:10.1017/S0022112073000911

25. Trowbridge JH, Geyer WR, Bowen MM, Williams AJ (1999) Near-bottom turbulence measurements in a partially mixed estuary: turbulent energy balance, velocity structure, and along-channel momentum balance. J Phys Oceanogr 29:3056-3072. doi:10.1175/1520-0485(1999)029<3056:NBTMIA>2.0.CO;2 